EPJ manuscript No.

(will be inserted by the editor)

\title{
Deuteron radial moments for renormalized chiral potentials
}

\author{
$\underline{\text { E. Ruiz Arriola }}^{a}$ and M. Pavon Valderrama \\ Departamento de Física Atómica, Molecular y Nuclear, Universidad de Granada, E-18071 Granada, Spain
}

December 9, 2018

\begin{abstract}
We calculate deuteron positive and negative radial moments involving any bilinear function of the deuteron S and D wave functions for renormalized OPE and TPE chiral potentials. The role played by the strong singularities of the potentials at the origin and the short distance insensitivity of the results when the potentials are fully iterated is emphasized as compared to realistic potentials.
\end{abstract}

PACS. $03.65 . \mathrm{Nk} 11.1013 .75$

Chiral dynamics has played an important role in the theoretical description of low energy hadronic reactions [1] and so far is the only known vestige of the underlying fundamental QCD theory of strong interactions in nuclear physics. There is a number of low energy theorems based on chiral symmetry which provide a quantitative and model independent insight into low energy processes involving pions and nucleons, due to the clear scale separation between nuclear physics and QCD. For compound systems which at low energies disclose their composite nature the theoretical description necessarily becomes very involved and probably dependent on arbitrary assumptions. On the contrary, for weakly bound systems such as the deuterium nucleus one expects important simplifications leading to a more scheme independent and possibly systematic description of these systems. This possibility motivated the introduction of Effective Field Theory (EFT) approaches 2 for nuclear physics based on the chiral symmetry of QCD, and the derivation of low energy theorems, as, for example, pion-deuteron scattering [3] (for comprehensive reviews see e.g. Ref. $4,5,6]$ ). In many cases most of the information needed for reactions involving the deuteron can be encoded by simple deuteron matrix elements.

Guided by earlier work 7.8 , we have proposed 9 , 10,11,12,13 to renormalize the NN interaction in a nonperturbative way, highlighting model independent long distance correlations among physical observables. In our approach the long distance chiral NN One Pion Exchange (OPE) and Two Pion Exchange (TPE) potentials, computed within perturbation theory in Refs. [14,15,16], are iterated to all orders in the Schrödinger equation very much in the spirit of the original Weinberg approach [2]. However, some subtleties are found 10 12,17, which im-

\footnotetext{
a Poster presented at QNP06 Madrid, 5-10 June 2006. Work supported by Spanish DGI and FEDER funds with grant no. BFM2002-03218, Junta de Andalucía grant No. FQM-225, and EU RTN Contract CT2002-0311 (EURIDICE)
}

pose strong constraints on the admisible short distance physics based on orthogonality, uniqueness and finiteness of the results.

In the ${ }^{3} S_{1}-{ }^{3} D_{1}$ channel, the relative proton-neutron state for negative energy is described by the coupled equations

$$
\left(\begin{array}{cc}
-\frac{d^{2}}{d r^{2}}+U_{s}(r) & U_{s d}(r) \\
U_{s d}(r) & -\frac{d^{2}}{d r^{2}}+\frac{6}{r^{2}}+U_{d}(r)
\end{array}\right)\left(\begin{array}{c}
u \\
w
\end{array}\right)=-\gamma^{2}\left(\begin{array}{c}
u \\
w
\end{array}\right)(1)
$$

Here $\gamma=\sqrt{M B}$, with $B$ the deuteron binding energy and $M$ the nucleon mass, $U(r)=M V(r)$ are the reduced potentials and $u(r)$ and $w(r)$ are S- and D-wave deuteron reduced wave functions respectively. At long distances they satisfy,

$$
\left(\begin{array}{c}
u \\
w
\end{array}\right) \rightarrow A_{S} e^{-\gamma r}\left(\eta\left[1+\frac{3}{\gamma r}+\frac{3}{(\gamma r)^{2}}\right]\right)
$$

where $\eta$ is the asymptotic $\mathrm{D} / \mathrm{S}$ ratio parameter and $A_{S}$ is the asymptotic normalization factor, which is such that the deuteron wave functions are normalized to unity. For conventions and numerical values of parameters we use Ref. 11,12 throughout.

In this work we report on the radial moments

$$
\begin{aligned}
\left\langle r^{n}\right\rangle_{u} & =\int_{0}^{\infty} r^{n} u(r)^{2} d r \\
\left\langle r^{n}\right\rangle_{w} & =\int_{0}^{\infty} r^{n} w(r)^{2} d r \\
\left\langle r^{n}\right\rangle_{u w} & =\int_{0}^{\infty} r^{n} u(r) w(r) d r
\end{aligned}
$$

for $-3 \leq n \leq 2$ which appear in many situations of interest, such as the calculation of the matter radius, the deuteron quadrupole moment and deuteron magnetic moment for the positive powers, as well as $\pi d$ and $K d$ elastic 

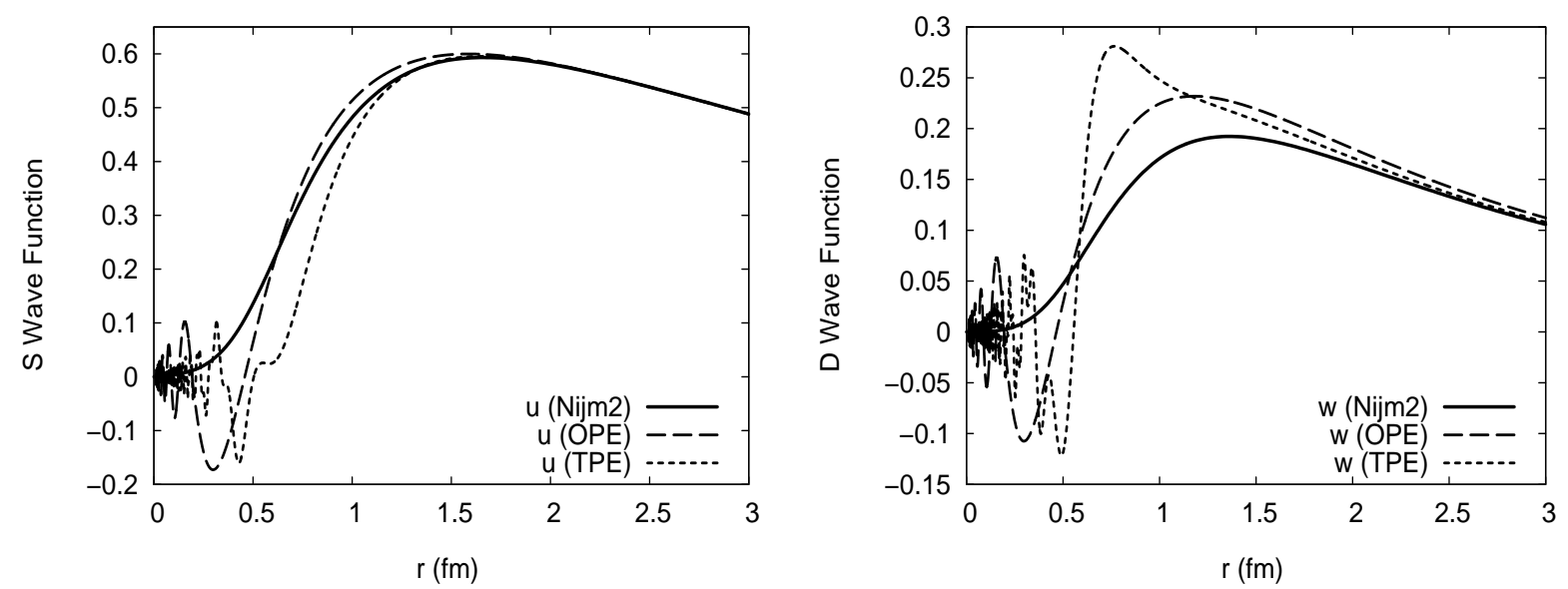

Fig. 1. The OPE and TPE deuteron wave functions, $u$ (left) and $w$ (right), as a function of the distance (in fm) compared to the Nijmegen II wave functions [22. The asymptotic normalization $u \rightarrow e^{-\gamma r}$ has been adopted and the asymptotic D/S ratio is taken $\eta=0.0256(4)$ in the TPE case (for OPE $\eta=0.026333$ ). We use the set IV of chiral couplings (see Ref. [12]).

scattering and neutral pion photoproduction, $\gamma d \rightarrow \pi^{0} d$, in the case of the negative powers.

An important issue is the finiteness of the negative radial moments, a topic which has been recently discussed for OPE [11 18, 19] and TPE [20. The remarkable finding is that chiral potentials [14]15], 16, when fully iterated, have an increasing number of finite inverse radial moments due to the near the origin singularities of the potential. They smoothen the short distance behaviour of the wave functions and hence improve the convergence of the inverse radial moments. This is in sharp contrast with perturbative approaches 21, for which the perturbative wave functions diverge at the origin 11 12 ${ }^{1}$, or conventional (regular) phenomenological potentials [22] where the Sand $\mathrm{D}$-wave short distance behaviour of the wave functions, $u \sim r$ and $w \sim r^{5}$ respectively, is enough to render $\langle 1 / r\rangle_{u}$ and $\left\langle 1 / r^{2}\right\rangle_{u}$ finite, but produce divergent higher inverse moments. We illustrate the situation below.

At distances much shorter than the pion Compton wavelength, the OPE potential behaves as

$$
\left(\begin{array}{ll}
U_{s}^{\mathrm{OPE}}(r) & U_{s d}^{\mathrm{OPE}}(r) \\
U_{s d}^{\mathrm{OPE}}(r) & U_{d}^{\mathrm{OPE}}(r)
\end{array}\right) \rightarrow \frac{1}{r^{3}}\left(\begin{array}{cc}
R_{s} & R_{s d} \\
R_{s d} & R_{d}
\end{array}\right)
$$

with $R_{s}=0, R_{s d}=2 \sqrt{2} R, R_{d}=4 R$ and $R=3 g_{A}^{2} M / 32 \pi f^{2}$ $(=1.07764 \mathrm{fm})$. This behaviour of the potential is strong enough to overcome the centrifugal barrier at short distances, thus modifying the usual short distance behaviour of the wave functions, which can schematically be written as

$$
u(r) \sim w(r) \sim\left(\frac{r}{R}\right)^{3 / 4} f\left(\frac{r}{R}\right)
$$

where $f(r / R)$ represents some linear combination of $\sin (4 \sqrt{R / r}), \cos (4 \sqrt{R / r})$ and $\exp (-4 \sqrt{2} \sqrt{R / r})$ (for a

\footnotetext{
1 This divergency holds for perturbations both on boundary conditions or on distorded (fully iterated) OPE waves.
}

complete analysis see Ref. [11]). The elimination of the diverging exponential fixes $\eta_{\mathrm{OPE}}=0.0263$. From this short distance behaviour of the wave functions, one finds that the $\langle 1 / r\rangle_{u}$ and $\left\langle 1 / r^{2}\right\rangle_{u}$ moments are finite for the OPE potential, while $\left\langle 1 / r^{3}\right\rangle_{u}$ and higher moments diverge, as it would happen for a regular potential.

The short distance behaviour of the TPE (NNLO) has been exploited in Ref. [12. The potential at short distances behaves as 14,15, 16

$$
\left(\begin{array}{ll}
U_{s}^{\mathrm{TPE}}(r) & U_{s d}^{\mathrm{TPE}}(r) \\
U_{s d}^{\mathrm{TPE}}(r) & U_{d}^{\mathrm{TPE}}(r)
\end{array}\right) \rightarrow \frac{1}{r^{6}}\left(\begin{array}{cc}
R_{s}^{4} & R_{s d}^{4} \\
R_{s d}^{4} & R_{d}^{4}
\end{array}\right)
$$

where

$$
\begin{aligned}
\left(R_{s}\right)^{4} & =\frac{3 g_{A}^{2}}{128 f^{4} \pi^{2}}\left(4-3 g_{A}^{2}+24 \bar{c}_{3}-8 \bar{c}_{4}\right) \\
\left(R_{s d}\right)^{4} & =-\frac{3 \sqrt{2} g_{A}^{2}}{128 f^{4} \pi^{2}}\left(-4+3 g_{A}^{2}-16 \bar{c}_{4}\right) \\
\left(R_{d}\right)^{4} & =\frac{9 g_{A}^{2}}{32 f^{4} \pi^{2}}\left(-1+2 g_{A}^{2}+2 \bar{c}_{3}-2 \bar{c}_{4}\right)
\end{aligned}
$$

and $\bar{c}_{i}=M c_{i}$ are the low energy chiral couplings appearing in $\pi N$ scattering. As in the OPE case, this potential is strong enough at short distances to modify the short distance behaviour of the wave function, which now reads

$$
\begin{aligned}
u(r) \sim w(r) \sim & C_{+}\left(r / R_{+}\right)^{3 / 2} f_{+}\left(r / R_{+}\right)+ \\
& C_{-}\left(r / R_{-}\right)^{3 / 2} f_{-}\left(r / R_{-}\right)
\end{aligned}
$$

where $R_{+}^{4}$ and $R_{-}^{4}$ are the eigenvalues of the matrix in Eq. (8), and $f_{ \pm}\left(r / R_{ \pm}\right)$represents a linear combination of $\sin \left(R_{ \pm}^{2} / 2 r^{2}\right)$ and $\cos \left(R_{ \pm}^{2} / 2 r^{2}\right)$ leaving $\eta_{\mathrm{TPE}}$ as a free parameter 12,20. From this short distance behaviour, the $\langle 1 / r\rangle_{u},\left\langle 1 / r^{2}\right\rangle_{u}$ and $\left\langle 1 / r^{3}\right\rangle_{u}$ radial moments are finite for the TPE potential, while higher moments diverge (although they would become finite for higher order potentials). 


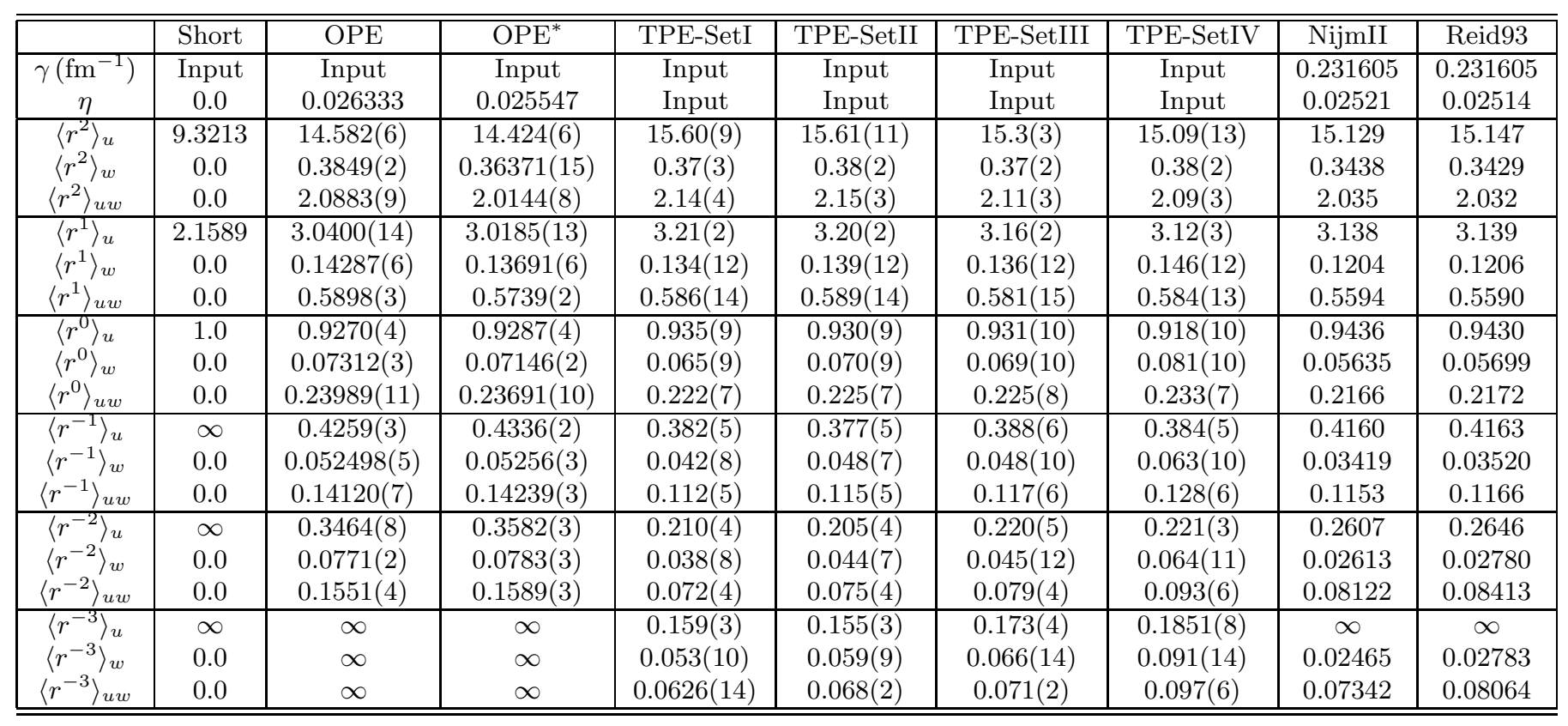

Table 1. Deuteron radial moments (in units of powers of fm). We consider the OPE and TPE potentials; in the case of the OPE potential we have taken $g_{\pi N N}=13.08$ (i.e. $g_{A}=1.29$, OPE) and $g_{A}=1.26\left(\mathrm{OPE}^{*}\right)$, while in the TPE case we show the results corresponding to the four set of chiral couplings considered along our previous works [12 20]. In the OPE case the error is estimated by varying the semiclassical matching radius [11 20] in the $0.1-0.2 \mathrm{fm}$ range, while in the TPE case the error comes from the experimental uncertainty of the $D / S$ ratio, $\eta=0.0256(4)$. TPE Sets I,II,II and IV refer to the chiral parameters, $c_{1}$, $c_{3}$ and $c_{4}$ of Refs. [26], [16], 24] and [25] respectively. Nijm II and Reid93 are calculated from Ref. [22] or taken from Ref. [27].

The wave functions for OPE and TPE as compared to the NijmII ones have been depicted in Fig. 11 The radial moments are tabulated in Table 1 As we see, and despite the very different behaviour at short distances between the deuteron wave functions corresponding to renormalized chiral potentials and to phenomenological potentials, the convergent moments are fairly similar (the $u+w$ combination works better) despite that NijmII and Reid93 contain no explicit TPE components. For inverse moments the trend improves clearly when going from OPE to TPE, which we interpret as a correct implementation of model independent long distance correlations generated by chiral symmetry and renormalization constraints.

\section{References}

1. T. E. O. Ericson and W. Weise, Pions and Nuclei. Oxford, UK: Clarendon (1988).

2. S. Weinberg, Phys. Lett. B 251, 288 (1990).

3. S. Weinberg, Phys. Lett. B 295, 114 (1992)

4. P. F. Bedaque and U. van Kolck, Ann. Rev. Nucl. Part. Sci. 52, 339 (2002)

5. D. R. Phillips, Czech. J. Phys. 52, B49 (2002)

6. D. R. Phillips, J. Phys. G 31, S1263 (2005)

7. D. W. L. Sprung, W. van Dijk, E. Wang, D. C. Zheng, P. Sarriguren, and J. Martorell, Phys. Rev. C49, 2942 (1994).

8. S. R. Beane, P. F. Bedaque, M. J. Savage and U. van Kolck, Nucl. Phys. A 700, 377 (2002) nucl-th/0104030
9. M. Pavon Valderrama and E. Ruiz Arriola, Phys. Lett. B 580, 149 (2004) nucl-th/0306069

10. M. Pavon Valderrama and E. Ruiz Arriola, Phys. Rev. C 70, 044006 (2004) nucl-th/0405057

11. M. Pavon Valderrama and E. Ruiz Arriola, Phys. Rev. C 72, 054002 (2005) nucl-th/0504067

12. M. P. Valderrama and E. R. Arriola, nucl-th/0506047

13. M. Pavon Valderrama and E. Ruiz Arriola, nucl-th/0507075

14. N. Kaiser, R. Brockmann and W. Weise, Nucl. Phys. A 625, 758 (1997)

15. J. L. Friar, Phys. Rev. C 60, 034002 (1999)

16. M. C. M. Rentmeester, R. G. E. Timmermans, J. L. Friar and J. J. de Swart, Phys. Rev. Lett. 82, 4992 (1999)

17. A. Nogga, R. G. E. Timmermans and U. van Kolck, Phys. Rev. C 72, 054006 (2005) nucl-th/0506005

18. A. Nogga and C. Hanhart, Phys. Lett. B 634, 210 (2006)

19. L. Platter and D. R. Phillips, Phys. Lett. B 641, 164 (2006)

20. M. P. Valderrama and E. R. Arriola, nucl-th/0605078

21. B. Borasoy and H. W. Griesshammer, Int. J. Mod. Phys. E 12, 65 (2003).

22. V. G. J. Stoks, R. A. M. Klomp, C. P. F. Terheggen and J. J. de Swart, Phys. Rev. C 49, 2950 (1994)

23. N. Fettes and U. G. Meissner, Nucl. Phys. A 693, 693 (2001) hep-ph/0101030

24. D. R. Entem and R. Machleidt, Phys. Rev. C66, 014002 (2002), nucl-th/0202039

25. D. R. Entem and R. Machleidt, Phys. Rev. C 68, 041001 (2003) nucl-th/0304018

26. P. Buettiker and U. G. Meissner, Nucl. Phys. A 668, 97 (2000) hep-ph/9908247 
27. J. J. de Swart, C. P. F. Terheggen and V. G. J. Stoks, nucl-th/9509032 